dations to treat CA UTIs only when they are symptomatic, the symptoms associated with CA UTI have not been clearly defined. Researchers at the University of Wisconsin Hospitals, Madison, reported on the findings of a study to determine the prevalence of signs and symptoms attributable to CA UTI and the relative contribution of CA UTI to nosocomial bloodstream infection (BSI).

In a prospective study, 1,497 newly catheterized patients were followed, and daily quantitative urine cultures and urine leukocyte counts were obtained. A detailed analysis was done of a subset of 1,024 patients, 89 of whom developed CA UTI with more than 103 colony-forming units per $\mathrm{mL}$, who did not have another potentially confounding site of infection besides the urinary tract.

There were 235 new cases of nosocomial CA UTI during the study period. More than $90 \%$ of the infected patients were asymptomatic; only 123 infections (52\%) were detected by patients' physicians using the hospital laboratory. In the subset analysis, there were no significant differences between patients with and without $\mathrm{CA}$ UTI in signs or symptoms commonly associated with UTI-fever, dysuria, urgency, or flank pain —or leukocytosis. Only 1 of the 235 episodes of CA UTI that were prospectively studied was unequivocally associated with secondary BSI.

CA UTIs are a major reservoir of antibiotic-resistant organisms in the hospital, but they are rarely symptomatic and infrequently cause BSI. Symptoms referable to the urinary tract, fever, or peripheral leukocytosis have little predictive value for the diagnosis of CA UTI.

FROM: Tambyah PA, Maki DG. Catheter-associated urinary tract infection is rarely symptomatic: a prospective study of 1,497 catheterized patients. Arch Intern Med 2000;160:678-682.

\section{Plague as Biological Weapon}

The Working Group on Civilian Biodefense has developed consensus-based recommendations for measures to be taken by medical and public health professionals following the use of plague as a biological weapon against a civilian population. The working group included 25 representatives from major academic medical centers and research, government, military, public health, and emergency-management institutions and agencies. The group developed a consensus statement supported by all working group members.

The statement concludes that an aerosolized plague weapon could cause fever, cough, chest pain, and hemoptysis, with signs consistent with severe pneumonia 1 to 6 days after exposure. Rapid evolution of disease would occur in the 2 to 4 days after symptom onset and would lead to septic shock, with high mortality without early treatment. Early treatment and prophylaxis with streptomycin or gentamicin or the tetracycline or fluoroquinolone classes of antimicrobials would be advised.

FROM: Inglesby TV, Dennis DT, Henderson DA, Bartlett JG, Ascher MS, Eitzen E, et al. Plague as a biologic weapon: Medical and public health management. Recommendations for Plague. JAMA 2000;283:2281-2290.

\section{Antimicrobial Susceptibility Testing of $P$ aeruginosa Isolates From CF Patients}

Pseudomonas aeruginosa is the most common pathogen infecting the lungs of patients with cystic fibrosis (CF). Improved antimicrobial chemotherapy has significantly increased the life expectancy of these patients. However, accurate susceptibility testing of $P$ aeruginosa isolates from CF sputum may be difficult, because the organisms are often mucoid and slow growing. Burns and coinvestigators, from the University of Washington, Seattle, reported on a study of $597 \mathrm{CF}$ isolates of $P$ aeruginos $a$ and examined the correlation of disk-diffusion and E-test (AB BIODISK, Solna, Sweden) results with a reference broth microdilution method. The rates of interpretive errors for 12 commonly used antipseudomonal antimicrobials were determined. The disk-diffusion method correlated well (zone diameter vs minimum inhibitory concentration [MIC]) for all of the agents tested. However, for mucoid isolates, correlation coefficients ( $\mathrm{r}$ values) for piperacillin, piperacillin-tazobactam, and meropenem were $<0.80$. The E-test correlation with reference broth microdilution results (MIC vs MIC) was acceptable for all of the agents tested, for both mucoid and nonmucoid isolates. Category interpretation errors were similar for the disk-diffusion and E-test methods, with 0.4 and $0.1 \%$, respectively, very major errors (false susceptibility) and 1.1 and $2.2 \%$ major errors (false resistance). Overall, both agar diffusion methods appear to be broadly acceptable for routine clinical use in susceptibility testing of $\mathrm{CF}$ isolates of $P$ aeruginosa.

FROM: Burns JL, Saiman L, Whittier S, Larone D, Krzewinski J, Liu $Z$, et al. Comparison of agar diffusion methodologies for antimicrobial susceptibility testing of Pseudomonas aeruginosa isolates from cystic fibrosis patients. J Clin Microbiol 2000;38:1818-1822. 